\title{
Study of the spatial resolution of low-material GEM tracking detectors
}

\author{
V.N.Kudryavtsev ${ }^{1,2}$, T.V.Maltsev ${ }^{1,2}$, and L.I.Shekhtman ${ }^{1,2, a}$ \\ ${ }^{1}$ Budker Institute of Nuclear Physics, 11.av.Lavrentiev, 630090, Novosibirsk, Russia \\ ${ }^{2}$ Novosibirsk State University, 2 Pirogova str., 630090, Novosibirsk, Russia
}

\begin{abstract}
The spatial resolution of GEM based tracking detectors has been simulated and measured. The simulation includes the GEANT4 based transport of high energy electrons with careful accounting for atomic relaxation processes including emission of fluorescent photons and Auger electrons and custom post-processing, including accounting for diffusion, gas amplification fluctuations, the distribution of signals on readout electrodes, electronics noise and a particular algorithm of the final coordinate calculation (center of gravity). The simulation demonstrates that a minimum of the spatial resolution of about $10 \mu \mathrm{m}$ can be achieved with strip pitches from $250 \mu \mathrm{m}$ to $300 \mu \mathrm{m}$. For larger pitches the resolution is quickly degrading reaching $80-100 \mu \mathrm{m}$ at a pitch of $500 \mu \mathrm{m}$. The spatial resolution of low-material triple-GEM detectors for the DEUTRON facility at the VEPP-3 storage ring is measured at the extracted beam facility of the VEPP-4M collider. The amount of material in these detectors is reduced by etching the copper of the GEMs electrodes and using a readout structure on a thin kapton foil rather than on a glass fibre plate. The exact amount of material in one DEUTRON detector is measured by studying multiple scattering of $100 \mathrm{MeV}$ electrons in it. The result of these measurements is X/X0 $=2.4 \times 10^{-3}$ corresponding to a thickness of the copper layers of the GEM foils of $3 \mu \mathrm{m}$. The spatial resolution of one DEUTRON detector is measured with $500 \mathrm{MeV}$ electrons and the measured value is equal to $35 \pm 1 \mu \mathrm{m}$ for orthogonal tracks.
\end{abstract}

\section{Introduction}

Triple-GEM tracking detectors can provide precise measurements of track positions in high rate environments [1]. These detectors can be produced with a low material budget that is important for precise tracking in medium and low energy particle and nuclear physics experiments [2]. Experimental data on the spatial resolution of the GEM detectors for relativistic charged particles lie in the range between $50 \mu \mathrm{m}$ and $100 \mu \mathrm{m}$ (see for example [3] and [4]) depending on the particular type of the readout structure, gas mixture, electronics, etc. The spatial resolution of this kind of detectors is determined by a combination of many factors, such as: statistics and ranges of primary deltaelectrons, transverse diffusion of electrons, configuration of the readout structure, noise of the readout electronics, fluctuations of the gas amplification, and the particular algorithm for the calculation of the track position. In order to determine the optimal set of parameters to get the best spatial resolution, we have started a systematic simulation and experimental study. The present paper presents preliminary results of the simulations of spatial resolution of the triple-GEM detectors with different layouts of the readout structure as well as experimental results, obtained with the detector produced for the DEUTRON facility at the VEPP-3 storage ring at the Budker INP [5].

\footnotetext{
a e-mail: L.I.Shekhtman@inp.nsk.su
}

\section{Simulation studies}

The simulation of the spatial resolution of the triple-GEM detectors was performed in two stages. At first, primary electrons with $1 \mathrm{GeV} / \mathrm{c}$ momentum perpendicular to the detector plane, and with randomly distributed initial coordinates in the detector plane are transported through the complete model of the detector, that includes the honeycomb cover with the drift electrode, the drift gap $(3 \mathrm{~mm})$, two transport gaps $(1.5 \mathrm{~mm})$, and the induction $(2 \mathrm{~mm})$ gas gap, three standard GEMs and the readout structure on a glass fibre plate (thick version of the detector, Fig. 1). In the simulation of the "thin" detector only $3 \mathrm{~mm}$ gas layer is left in the first stage. The energy depositions coming from other detector elements, such as the honeycomb with the drift electrode and the GEMs are removed. However in the second stage we take into account that the ionisation drifts through all GEMs and the induction gap, and distribute the signals using realistic readout strip plane configuration. After the recording of all energy depositions in the drift gap, the second stage is started that includes the introduction of the electron diffusion, gas gain fluctuations, the distribution of the signal between the readout strips, accounting for the electronic noise, and the calculation of the measured track position with the centre of gravity (COG) method.

Three types of the readout plane layouts were used in the simulation. The first layout contains parallel strips with their width equal to the pitch (no gaps between strips) 


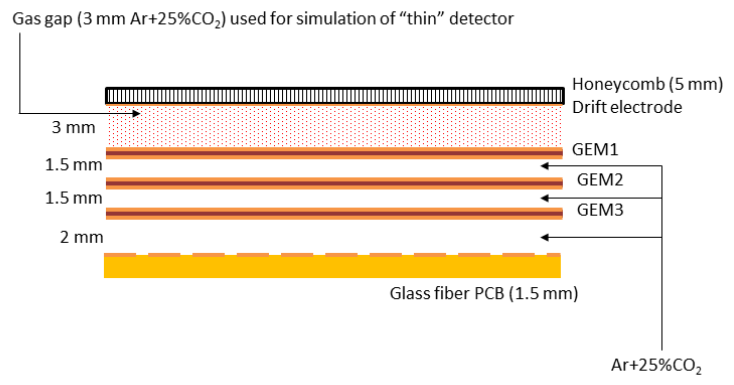

Figure 1. Schematic view of the model of "thick" detector. The "thin" detector was simulated with a single $3 \mathrm{~mm}$ layer of gas mixture (marked with pink background in the figure).

and each of these strips is read out. The second layout also contains parallel strips but only each second strip is read out. In the third layout that also contains parallel strips, only each third strip is read out.

These three layouts correspond to the cases where the full signal is induced on the strips (case 1), half of the signal is induced on the strips (case 2), and one third of the signal is induced on the strips (case 3). Case two and three correspond to the detectors with two layer readout planes with straight strips and stereo strips like in the detectors for the DEUTRON facility [6] and in the detectors for the tagging system of the KEDR experiment at the VEPP-4M [4]. In these detectors only part of the signal is induced on the straight strips used for the precise position measurement in one direction. The rest of the signal is induced at the stereo strips that are necessary for the position measurement in the perpendicular direction and were not used in the present study. In the detectors for the tagging system of the KEDR due to a mistake during manufacturing of the readout planes the signal induction is very non-uniform and only one third of the signal is in average induced on the straight strips.

At first the difference between the resolution of the "low material" detector and the thick detector was checked for the first layout of the readout plane. In Fig. 2 the resolution as a function of the strip pitch is presented for different values of the signal to noise ratio. The figure demonstrates that the effect of the low material on the spatial resolution is marginal and is only pronounced at strip pitches smaller than $300 \mu \mathrm{m}$. All further simulation results are obtained for the thick detector layout.

In Fig. 3 the resolution as a function of the strip pitch is shown for the readout plane of the third type and for several values of the signal to noise ratio. The experimental result from [4] for the detector of the tagging system of KEDR is also shown in the figure. The COG method used in this simulation for the calculation of the track position involved always three strips around the maximum of the charge distribution. Results of the simulations for the readout structure of the second type are presented in Fig. 4. The so called "flexible" COG method was used in this case, with the number of strips involved depending

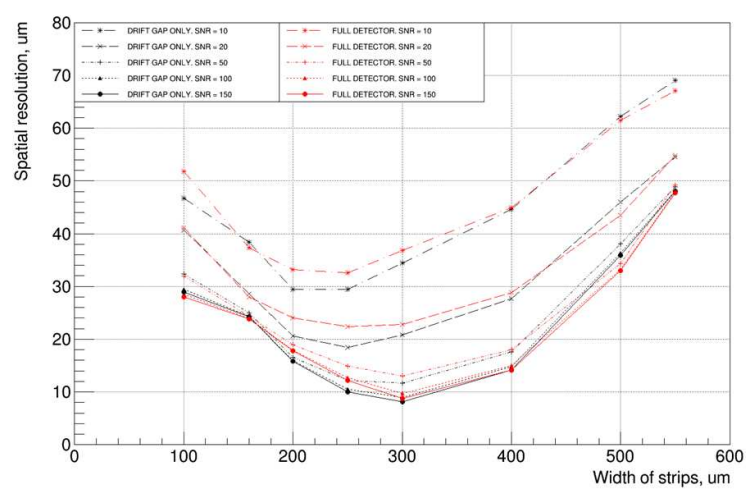

Figure 2. Comparison of spatial resolution of "low-material" detector (only $3 \mathrm{~mm}$ drift gap, black points and curves) and regular detector with all the materials (red points and curves).

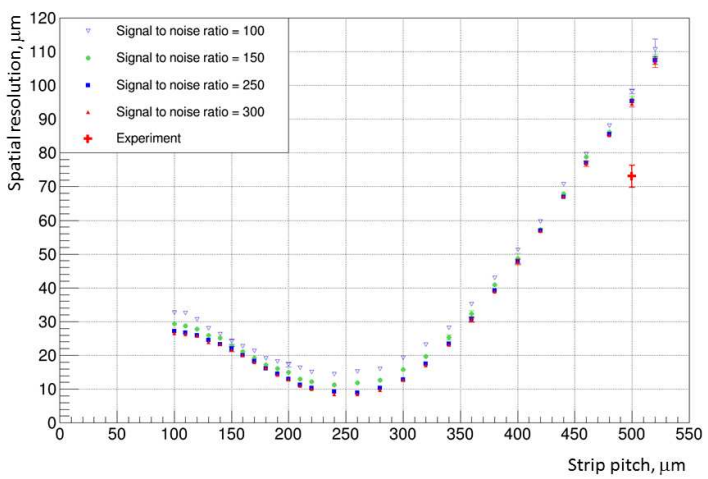

Figure 3. Spatial resolution as a function of the strip pitch for the readout structure of the KEDR TS detectors [4]. The experimental point is taken from [4].

on the relationship between the charge cluster size and the strip pitch. An increase of the number of strips involved in the COG calculation explains the sudden changes in the resolution around and below $100 \mu \mathrm{m}$ strip pitch. From the figures we can see that the best resolution of 10-15 $\mu \mathrm{m}$ can be achieved for a strip pitch of $250 \mu \mathrm{m}$. For larger pitches the resolution degrades and the results of the simulation are worse than the experimental results (Fig. 3 and also see below).

\section{Experimental results}

The amount of material and the spatial resolution of the triple-GEM detectors for the DEUTRON photon tagging system (PTS) [6] (Fig. 5) were measured at the extracted electron beam facility at the VEPP-4M storage ring [7]. Three detectors were installed at the test bench. The first and the second were "thin" detectors for the DEUTRON PTS with the dimensions of the sensitive area of $160 \times 40$ 


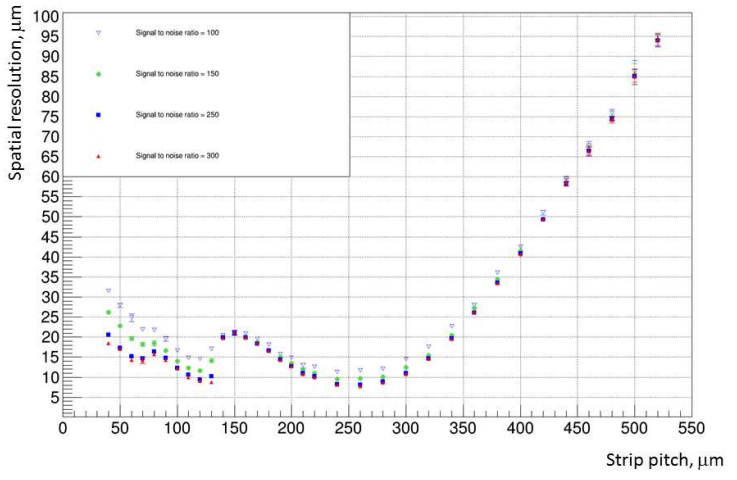

Figure 4. Spatial resolution as a function of the strip pitch for the readout structure of the DEUTRON PTS detectors [6] (see below).

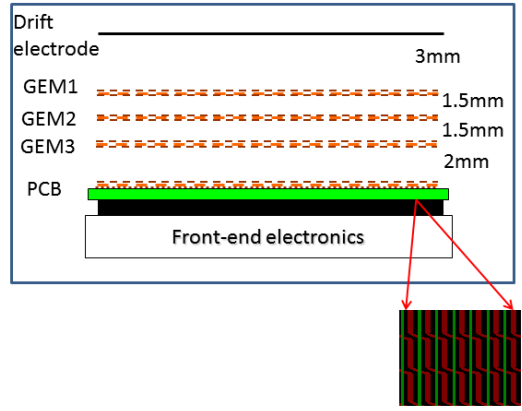

Figure 5. DEUTRON PTS detector structure and schematic view of the PCB design.

$\mathrm{mm}^{2}$ and the third was the detector for the KEDR TS with a sensitive area of $128 \times 100 \mathrm{~mm}^{2}$.

The readout strip structure of the DEUTRON PTS detectors is produced on a $50 \mu \mathrm{m}$ thick Kapton foil and all copper layers on the GEM foils and the readout flexible PCB are reduced as much as possible to decrease the amount of material. The readout flex is produced in two layer technology and the strip pitch in each layer is equal to $0.5 \mathrm{~mm}$. The strips of the stereo layer are shifted by $250 \mu \mathrm{m}$ with respect to the strips of the straight layer. The amount of material in the detector was determined by measuring the multiple scattering of $100 \mathrm{MeV}$ electrons in the experimental set-up shown in Fig. 6.

Due to the multiple scattering the track angles were changing after passing through the second detector in the setup. The distribution of the differences between the track angles after the second detector and before the second detector is shown in Fig. 7. Its width corresponds to an

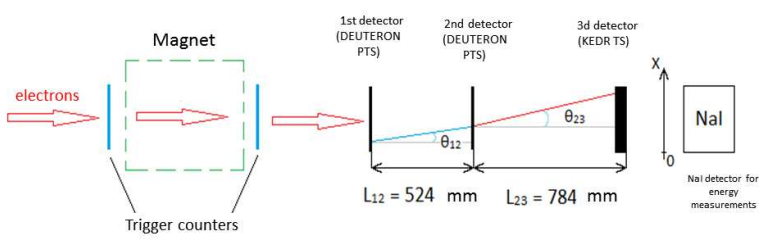

Figure 6. Schematic view of the set-up for the measurement of the amount of material in the detector.

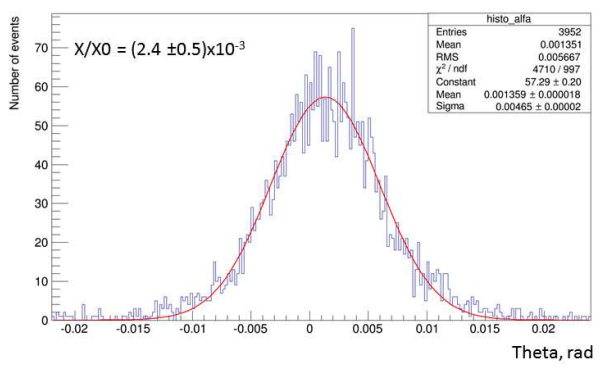

Figure 7. Angular distribution of tracks of $100 \mathrm{MeV}$ electrons scattered in the 2nd detector (see Fig. 6).

amount of material of $\sim 2.4 \times 10^{-3} \mathrm{X} 0$. This value corresponds to a copper thickness on the GEM foils and the PCB of $\sim 3 \mu \mathrm{m}$.

The spatial resolution of the $2^{\text {nd }}$ detector was measured with a similar setup (Fig. 6), but the distances between the detectors were reduced to $\sim 75 \mathrm{~mm}$ and the beam energy was increased to $500 \mathrm{MeV}$. The first and the third detector were used for tracking. Figure 8 demonstrates the resolution as a function of the track angle after correction for multiple scattering and for the limited resolution of the tracking detectors. The error bars in the figure represent the statistical errors. The curve in the figure is calculated using the formula of the quadratic sum of the resolution for orthogonal tracks and the track projection to the detector plane: $\sigma=\sqrt{\left.\sigma_{0}^{2}+(L * \operatorname{tg}(\alpha))^{2} / 12\right)}$, where $\sigma_{0}$ is equal to $35 \mu \mathrm{m}$ and $\mathrm{L}$ is $3 \mathrm{~mm}$, the thickness of the drift gap.

\section{Conclusions}

Simulation studies show that an ultimate resolution of 10$15 \mu \mathrm{m}$ can be achieved for orthogonal tracks of high en- 
ergy electrons in triple-GEM detectors operating with a gas mixture of $\mathrm{Ar}-25 \% \mathrm{CO}_{2}$ with a strip readout structure at a pitch of $250 \mu \mathrm{m}$. At larger strip pitches the simulation shows a systematically worse resolution than the measurements that will require more studies.

The measurements demonstrate that a spatial resolution of the triple-GEM detector of $35 \pm 1 \mu \mathrm{m}$ can be achieved for a strip pitch of $0.5 \mathrm{~mm}$.

\section{Acknowledgements}

This study was supported by the Russian Science Foundation (project N 14-50-00080).

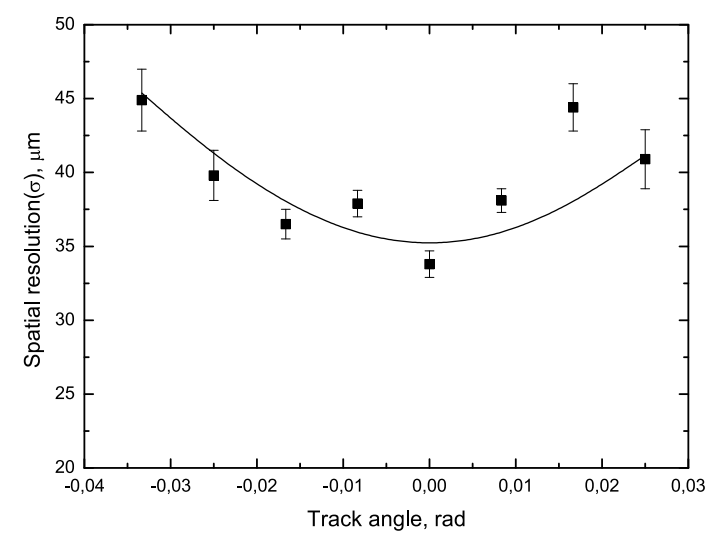

Figure 8. Spatial resolution as a function of the track angle.

\section{References}

[1] Sauli F., Sharma A. Micropattern Gaseous Detectors, Annu.Rev.Nucl.Part.Sci. 1999. 49. P.341-88.

[2] A. Bondar, et.al, Nucl. Instrum. and Methods., A556, (2006), P.495-497.

[3] B.Ketzer, et.al., IEEE Transactions on Nuclear Science V.49 II, N.5 (2002), P.2403-2410.

[4] V M Aulchenko, et.al., Journal of Instrum. 6, P07001 (2011).

[5] Shestakov, Yu V., et al., PHYSICS OF PARTICLES AND NUCLEI, 45, n.1 (2014), P.338-340.

[6] V N Kudryavtsev, et.al., Journal of Instrum., 9, C09024 (2014).

[7] G N Abramov, et.al., Journal of Instrum., 9, C08022 (2014). 